\title{
Investigation of Medium Chain Fatty Acid Feed Supplementation for Reducing Salmonella Typhimurium Colonization in Turkey Poults
}

\author{
Nicholas P. Evans, ${ }^{1, *}$ David A. Collins, ${ }^{1}$ Frank William Pierson,, Hassan M. Mahsoub,, \\ Nammalwar Sriranganathan, Mike E. Persia, Theodore Peter Karnezos, \\ Michael D. Sims, and Rami A. Dalloul ${ }^{4}$
}

\begin{abstract}
Studies indicate that persistent Salmonella colonization occurs in poultry that are infected early in life, leading to both food safety and public health concerns. Development of improved preharvest Salmonella management strategies is needed to reduce poultry product contamination. The objective of this study was to evaluate the efficacy of a product containing medium chain fatty acids (MCFA) for reducing early Salmonella colonization in turkey poults. Day-of-hatch turkeys were provided a standard starter diet supplemented with MCFA at 0 (negative and positive controls), 1.5, 3, 4.5, or $6 \mathrm{lbs} /$ ton of feed. Positive control and MCFA treated birds were also crop-gavaged with $10^{8}$ colony forming units (CFU) of bioluminescent Salmonella Typhimurium. Gastrointestinal tissue samples were collected at 3 days postinoculation for bioluminescence imaging (Meckel's diverticulum to the cloaca) and selective enumeration (cecal contents). Quantification of bioluminescence indicated that the 4.5 and $6 \mathrm{lbs} /$ ton MCFA groups had significantly less colonization than the positive control group $\left(p=0.0412\right.$ and $p<0.0001$, respectively). Similarly, significantly lower numbers $\left(1-\log _{10} \mathrm{CFU} / \mathrm{g}\right.$ reduction) of Salmonella were observed in the ceca of the $6 \mathrm{lbs} /$ ton MCFA group compared to the positive control group ( $p=0.0153)$. These findings indicate that incorporation of MCFA in turkey diets can significantly reduce early Salmonella colonization. In addition, this study highlights the utility of bioluminescence imaging as a screening methodology for assessing the efficacy of treatments that may reduce Salmonella in poultry.
\end{abstract}

Keywords: medium chain fatty acids, Salmonella, turkey, bioluminescence, food safety

\section{Introduction}

$\mathbf{I}$ N THE UNited States, an estimated 1.2 million human salmonellosis cases occur annually and are predominately associated with the consumption of contaminated food products (Scallan et al., 2011; Jackson et al., 2013). Foodborne illness surveillance has linked poultry products to multiple Salmonella outbreaks in humans (Gould et al. 2013).
Although contamination of poultry meat with Salmonella can be attributed to a number of factors, there remains a strong relationship between the gastrointestinal colonization of poultry and product contamination risk (Rasschaert et al., 2008; Evans et al., 2015).

Poultry are susceptible to developing persistent Salmonella gastrointestinal colonization if they become infected early in life (Gast and Beard, 1989; Gast and Holt, 1998; Van

\footnotetext{
${ }^{1}$ Department of Population Health Sciences, Virginia-Maryland College of Veterinary Medicine, Virginia Polytechnic and State University, Blacksburg, Virginia.

${ }^{2}$ Department of Poultry Production, Faculty of Agriculture, Alexandria University, Alexandria, Egypt.

${ }^{3}$ Department of Biomedical Sciences and Pathobiology, Virginia-Maryland College of Veterinary Medicine, Virginia Polytechnic and State University, Blacksburg, Virginia.

${ }^{4}$ Department of Animal and Poultry Sciences, College of Agriculture and Life Sciences, Virginia Polytechnic and State University, Blacksburg, Virginia.

${ }_{5}^{5}$ PMI Nutritional Additives ${ }^{\mathrm{TM}}$, Shoreview, Minnesota.

${ }^{6}$ Virginia Diversified Research Corporation, Harrisonburg, Virginia.

*Current address: PMI Nutritional Additives ${ }^{\mathrm{TM}}$, Shoreview, Minnesota.
}

(C) Nicholas P. Evans et al. 2017; Published by Mary Ann Liebert, Inc. This is an Open Access article distributed under the terms of the Creative Commons Attribution License, which permits unrestricted use, distribution, and reproduction in any medium, provided the original work is properly cited. 
Immerseel et al., 2004b). These birds remain colonized for the duration of the production cycle and provide a reservoir for the continued spread of Salmonella throughout the flock (Gast and Beard, 1989; Gast and Holt, 1998; Van Immerseel et al., 2004b). The stress associated with transport to the processing plant can then induce fecal shedding and lead to raw product contamination (Mulder, 1995; Marin and Lainez, 2009; Evans et al., 2015). Therefore, treatments that reduce early Salmonella colonization may have an impact on overall flock carriage levels and may help reduce product contamination.

Medium chain fatty acids (MCFA; C6-C12) have recently been investigated as a preharvest treatment for controlling Salmonella during poultry production. These molecules are more bactericidal than short chain fatty acids $(\leq \mathrm{C} 4)$ which are routinely used to combat Salmonella (Kwon and Ricke, 1998; Nakai and Siebert, 2003; Van Immerseel et al., 2003, 2006; Skrivanova et al., 2006). Interestingly, MCFA also suppress the expression of the invasion genes, hilA and hilD, in Salmonella and decrease intestinal epithelial cell invasion (Van Immerseel et al., 2004a; Kollanoor-Johny et al., 2012). Studies in both layers and broilers show that MCFA can reduce Salmonella colonization in the gastrointestinal tract and in other tissues (Van Immerseel et al., 2004a; Kollanoor-Johny et al., 2009, 2012; Upadhyaya et al., 2015). Potentially, preharvest MCFA treatment could have an impact on product contamination by reducing Salmonella colonization during production. However, additional studies are needed to further evaluate the efficacy of MCFA for combating Salmonella in not only chickens but also in turkeys.

Our laboratory has developed new methods for evaluating Salmonella gastrointestinal colonization. By inoculating birds with a bioluminescent Salmonella Typhimurium LT2 isolate and imaging with the In Vivo Imaging System (IVIS ${ }^{\circledR}$; PerkinElmer), we can view colonization in the gastrointestinal tract and investigate various treatment strategies. One advantage of the IVIS is the ability to quantify bioluminescence which is directly related to the levels of the target bacteria present (Karsi et al., 2008; Foucault et al., 2009). The objective of this study was to evaluate the efficacy of a product containing a combination of MCFA (C6-C12) for reducing early Salmonella colonization in turkey poults.

\section{Materials and Methods}

\section{Animals}

All animal experiments were approved by the Virginia Tech Institutional Animal Care and Use Committee. Upon arrival, 36 day-of-hatch female turkey poults (Aviagen Turkeys, Inc., Lewisburg, WV) were randomly assigned into six groups and placed into individual isolator cages $\left(n=5-7\right.$, with $340 \mathrm{~cm}^{2} /$ bird). To ensure birds were Salmonella negative at the start of the study, pooled cecal droppings were collected from each group and tested with the DNAble Molecular Detection Kit for Salmonella (DF-026) using Extraction Set 1 (EnviroLogix, Inc., Portland, ME) as described previously (Evans et al., 2015).

\section{Bioluminescent Salmonella culture and inoculum preparation}

A bioluminescent Salmonella enterica serovar Typhimurium LT2 isolate was used for this study. The isolate contains the expression vector pNSTrcD with bioluminescent luciferase cassette $(\mathrm{CDABE})$ and chloramphenicol resistance gene
(Seleem et al., 2007a, b; Seleem et al., 2008a, b). The transformed isolate was streaked onto tryptic soy agar (TSA; BD $\mathrm{Difco}^{\mathrm{TM}}$, Sparks, MD) and incubated overnight at $37^{\circ} \mathrm{C}$. For preparation of frozen inoculum stocks, a single bright colony was selected from a TSA plate and used to inoculate $20 \mathrm{~mL}$ of tryptic soy broth (TSB; BD Difco) in $50 \mathrm{~mL}$ conical tubes (Fisher Scientific, Hampton, NH). Tubes were then placed in a shaking $(175 \mathrm{rpm})$ incubator overnight at $37^{\circ} \mathrm{C}$. Cultures were pelleted by centrifugation at $2000 \times g$ for $15 \mathrm{~min}$. Pellets were washed twice in sterile phosphate-buffered saline (PBS; Fisher Scientific), pooled, and frozen at $-80^{\circ} \mathrm{C}$ with a final glycerol concentration of $20 \% \mathrm{v} / \mathrm{v}$. One week later, an aliquot of the frozen stock was thawed and streaked onto TSA and TSA $+5 \%$ sheep blood (Remel ${ }^{\mathrm{TM}}$, San Diego, CA). Plates were incubated overnight at $37^{\circ} \mathrm{C}$ to confirm purity. A plate count was performed to determine the concentration of Salmonella in the prepared stocks. In addition, a minimum inhibitory concentration (MIC) assay (Sensititre ${ }^{\mathrm{TM}}$; Thermo Scientific, Waltham, MA) was carried out by the Clinical Bacteriology Lab at the Virginia-Maryland College of Veterinary Medicine. The isolate was found to be resistant to $0.25 \mu \mathrm{g} / \mathrm{mL}$ of ceftiofur (chloramphenicol was not included in the panel). Chloramphenicol resistance was confirmed by growing the isolate overnight at $37^{\circ} \mathrm{C}$ on TSA plates supplemented with $30 \mu \mathrm{g} / \mathrm{mL}$ chloramphenicol (Fisher Scientific).

\section{Salmonella challenge}

Before challenge, each group of birds (day-of-hatch) was provided access to $82.5 \mathrm{~g}$ of $\mathrm{HydroGel}^{\mathrm{TM}}\left(\mathrm{ClearH}_{2} \mathrm{O}\right.$,

Table 1. Diet Composition of the Standard Starter Diet Fed to Turkey Poults

\begin{tabular}{|c|c|}
\hline Ingredient & (\%) Composition \\
\hline Corn & 44.23 \\
\hline Soybean meal (48\%) & 43.86 \\
\hline Poultry byproduct meal & 5.00 \\
\hline Soy oil & 0.99 \\
\hline Salt $(\mathrm{NaCl})$ & 0.19 \\
\hline Sodium bicarbonate & 0.20 \\
\hline DL-Methionine & 0.30 \\
\hline Lysine $\bullet \mathrm{HCl}$ & 0.26 \\
\hline Limestone & 1.09 \\
\hline Dicalcium phosphate & 3.02 \\
\hline Choline chloride $(60 \%)$ & 0.10 \\
\hline Vitamin/mineral premix ${ }^{a}$ & 0.75 \\
\hline \multicolumn{2}{|l|}{ Calculated composition } \\
\hline $\mathrm{ME}(\mathrm{kcal} / \mathrm{kg})$ & 2850 \\
\hline Crude protein $(\%)$ & 28.12 \\
\hline Calcium $(\%)$ & 1.40 \\
\hline Available P (\%) & 0.75 \\
\hline Dig Lys $(\%)$ & 1.62 \\
\hline Dig Met (\%) & 0.66 \\
\hline Dig Thr $(\%)$ & 0.97 \\
\hline Dig Met+Cys (\%) & 1.05 \\
\hline
\end{tabular}

${ }^{\text {a }}$ Provided the following per $\mathrm{kg}$ vitamin/mineral premix: cobalt $34 \mathrm{ppm}$; copper $540 \mathrm{ppm}$; iodine $134 \mathrm{ppm}$; iron $6750 \mathrm{ppm}$; manganese $8580 \mathrm{ppm}$; zinc $6500 \mathrm{ppm}$; vitamin A 881,848 IU/kg; vitamin D3 295,419 ICU/kg; vitamin E $220 \mathrm{IU} / \mathrm{kg}$; vitamin B12 $0.9 \mathrm{mg} / \mathrm{kg}$; menadione $154 \mathrm{mg} / \mathrm{kg}$; riboflavin $551 \mathrm{mg} / \mathrm{kg}$; D-pantothenic acid $811 \mathrm{mg} / \mathrm{kg}$; niacin $2646 \mathrm{mg} / \mathrm{kg}$; and choline $51,030 \mathrm{mg} / \mathrm{kg}$.

P, Phosphorus; Lys, lysine; Met, methionine; Thr, threonine; Met+Cys, methionine and cystine. 
Westbrook, ME) mixed with $18 \mathrm{~g}$ of standard starter diet (Table 1) containing a MCFA product (Nuscience Group, Ghent, Belgium) at 0 (negative and positive controls), 1.5, 3, 4.5 , or $6 \mathrm{lbs} / \mathrm{ton}$ of feed $(0,0.75,1.5,2.25,3 \mathrm{~g} / \mathrm{kg})$ for $1 \mathrm{~h}$. Birds were then fasted for $2 \mathrm{~h}$ during which time a frozen stock of bioluminescent Salmonella was thawed on ice and diluted in sterile PBS. Birds were either crop-gavaged with $1 \mathrm{~mL}$ of $1 \times 10^{8}$ colony forming units $(\mathrm{CFU}) / \mathrm{mL}$ of bioluminescent Salmonella (positive control and MCFA treated groups) or $1 \mathrm{~mL}$ of sterile PBS (negative control). Birds were then provided a standard starter feed (Table 1) containing the corresponding MCFA treatment as described above and water ad libitum for the duration of the study.

\section{Imaging of gastrointestinal samples}

Turkeys were humanely euthanized with $\mathrm{CO}_{2}$ at 3 days postinoculation (PI) for bioluminescence imaging. Gastrointestinal tissue samples were collected from Meckel's diverticulum to the cloaca. Samples were placed into containers premoistened with sterile PBS to prevent drying and kept warm in a $37^{\circ} \mathrm{C}$ incubator. The samples were opened longitudinally just before imaging and placed onto a black low-background Lexan ${ }^{\mathrm{TM}}$ sheet (PerkinElmer, Waltham, MA). An IVIS SpectrumCT (PerkinElmer) was used to quantify bioluminescence. After a 1-min incubation on the imaging stage at $37^{\circ} \mathrm{C}$, images were taken with a specimen height of $1.5 \mathrm{~cm}$. Exposure and binning were set accordingly to keep the bioluminescence values within 600-60,000 counts (relative luminescence units) as specified by the manufacturer. Regions of interest (ROI) were overlaid $(22 \times 22 \mathrm{~cm})$ on each image, and total flux (photons/s) was recorded for each sample.

\section{Salmonella enumeration}

Salmonella levels in the ceca were enumerated for each bird using a modified plate count procedure and then compared by treatment (Evans et al., 2015). Briefly, cecal contents were collected and weighed after imaging. Samples were diluted $1: 10 \mathrm{w} / \mathrm{v}$ in sterile buffered peptone water (BPW; BD Difco) and then serially diluted (1:10) in PBS. From each dilution, $10 \mu \mathrm{L}$ drops were plated in quadruplicate onto TSA plates supplemented with $30 \mu \mathrm{g} / \mathrm{mL}$ chloramphenicol and $0.25 \mu \mathrm{g} / \mathrm{mL}$ ceftiofur (Fisher Scientific). Plates were incubated for $10 \mathrm{~h}$ (colony diameter: $1-2 \mathrm{~mm}$ ) at $41^{\circ} \mathrm{C}$

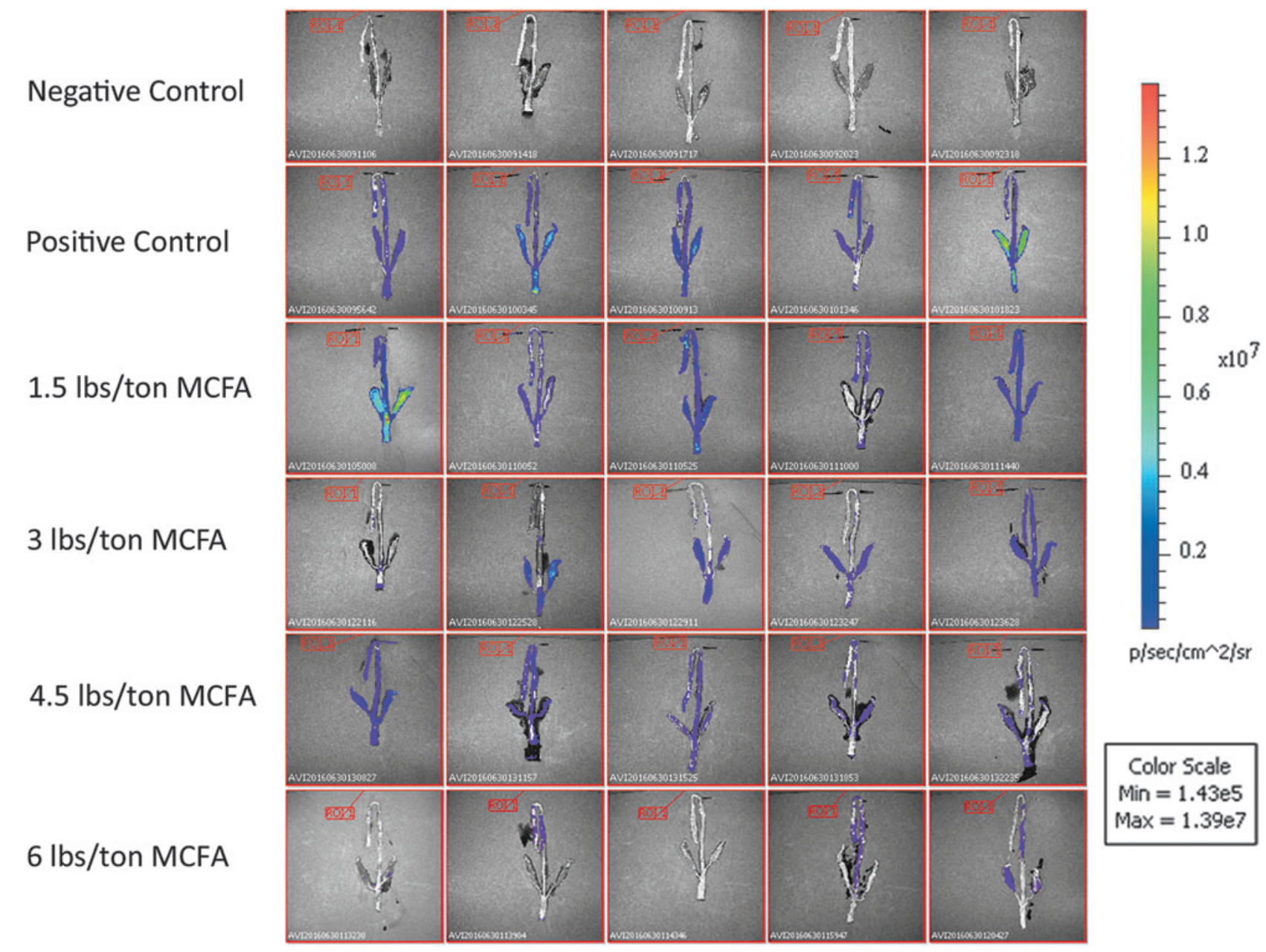

FIG. 1. Images of gastrointestinal samples (Meckel's diverticulum to cloaca) with pseudocolor overlays representing the amount of light produced by bioluminescent Salmonella Typhimurium ( 3 days PI). The color bar indicates the amount of bioluminescence (surface radiance: $\mathrm{p} / \mathrm{s} / \mathrm{cm}^{2} / \mathrm{sr}$ ) detected during imaging. ROI were overlaid on each image to quantify the total flux (photons/s) for each sample. MCFA, medium chain fatty acids; PI, postinoculation; ROI, regions of interest. 
to approximate turkey body temperature $\left(40.6-41.5^{\circ} \mathrm{C}\right)$. Following incubation, all plates were inspected with the IVIS SpectrumCT to confirm that only bioluminescent colonies were present, and $\mathrm{CFU} / \mathrm{g}$ were calculated for each sample.

\section{Statistical analysis}

Analysis of both bioluminescence and enumeration data was conducted using JMP Pro 11 (SAS Institute, Inc., Cary, $\mathrm{NC}$ ). Bioluminescence (photons/s) and enumeration (CFU/g) data were reported as the $\log _{10}$ mean for each treatment \pm standard error of the $\log _{10}$ mean. Data were analyzed as a one-way analysis of variance (ANOVA). When the model was significant $(p \leq 0.05)$, a post-hoc analysis (Least Squares [LS] Means Student's $t$-test) was performed.

\section{Results}

\section{Bioluminescent Salmonella imaging}

Imaging of bioluminescent Salmonella was conducted on intestinal tissues from Meckel's diverticulum to the cloaca at 3 days PI. A pseudocolor overlay representing the bioluminescent light produced by Salmonella colonizing the intestinal tract is shown in Figure 1. Visually, bioluminescence appeared to be lowest in the negative control group and highest in the positive control group. Light levels also appeared to be progressively reduced as the MCFA concentration increased. The highest levels of bioluminescence were typically observed in the ceca of infected birds, but bioluminescence was also present throughout the gastrointestinal samples. Total flux (photons/s) was quantified for each sample ROI and compared by treatment group (Fig. 2). The ANOVA analysis was significant $(p<0.0001)$, and the post-hoc LS Means Student's $t$-test indicated that both the $4.5 \mathrm{lbs} / \mathrm{ton}$ (7.9 $\log _{10}$ photons/s \pm 0.10$)$ and $6 \mathrm{lbs} /$ ton $\left(7.5 \log _{10}\right.$ photons/ $\mathrm{s} \pm 0.11)$ MCFA groups had significantly less ( $p=0.0412$ and $p<0.0001$, respectively) bioluminescence than the positive control group (8.4 $\log _{10}$ photons $\left./ \mathrm{s} \pm 0.15\right)$. The $6 \mathrm{lbs} /$ ton MCFA group was also significantly lower $(p=0.0276)$ than

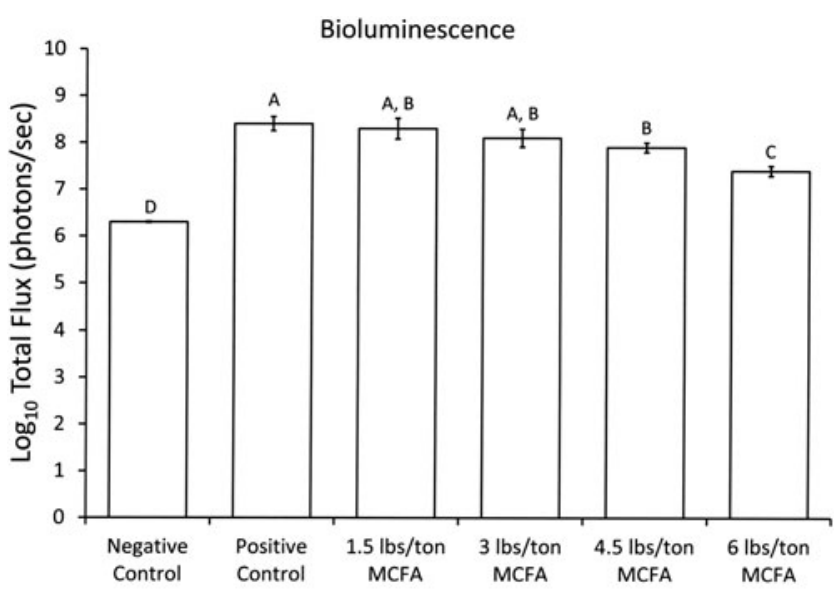

FIG. 2. Salmonella bioluminescence in gastrointestinal samples (Meckel's diverticulum to cloaca) from control and MCFA treated poults ( 3 days PI). Error bars represent standard error of the $\log _{10}$ mean for each treatment group. Treatments not connected by the same letter are significantly different $(p \leq 0.05)$. MCFA, medium chain fatty acids; PI, postinoculation.

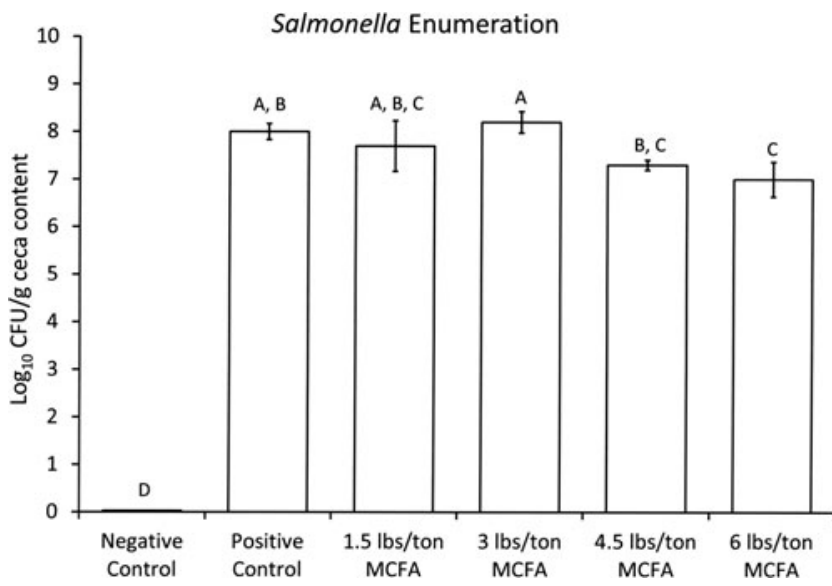

FIG. 3. Salmonella enumeration of cecal contents from control and MCFA treated poults (3 days PI). Error bars represent standard error of the $\log _{10}$ mean for each treatment group. Treatments not connected by the same letter are significantly different $(p \leq 0.05)$. MCFA, medium chain fatty acids; PI, postinoculation.

the $4 \mathrm{lbs} /$ ton MCFA group. There were no significant differences among the 1.5, 3 , and $4 \mathrm{lbs} /$ ton MCFA groups.

\section{Salmonella enumeration}

Samples collected from the ceca were quantified by plate count (Fig. 3). As expected, no Salmonella was recovered from birds in the negative control group. However, Salmonella was recovered from all other birds and ranged between 6.0 and $9.3 \log _{10} \mathrm{CFU} / \mathrm{g}$. The ANOVA analysis was significant $(p<0.0001)$, and the post-hoc test (LS Means Student's $t$-test) indicated that the $6 \mathrm{lbs} /$ ton MCFA $\left(7.0 \log _{10} \mathrm{CFU} / \mathrm{g} \pm\right.$ $0.37)$ group had a significant reduction $(p=0.0153)$ in Salmonella ( $1-\log _{10} \mathrm{CFU} / \mathrm{g}$ reduction) compared to the positive control group (8.0 $\left.\log _{10} \mathrm{CFU} / \mathrm{g} \pm 0.17\right)$.

\section{Discussion}

Poultry are susceptible to Salmonella infections during the first few days of life and begin developing greater resistance thereafter (Gast and Beard, 1989). Birds infected immediately after hatch have more persistent colonization and higher levels of fecal shedding than birds infected at 1 week posthatch (Gast and Beard, 1989; Van Immerseel et al., 2004b). Preharvest management strategies that effectively decrease early carriage levels may reduce the spread of Salmonella within a flock (Gast and Beard, 1989). Presumably, this would also help reduce product contamination. Therefore, the objective of this study was to evaluate the efficacy of a MCFA product for reducing early Salmonella colonization in turkey poults.

A novel bioluminescent Salmonella infection model was used to assess MCFA efficacy. One benefit of this model is the ability to screen treatments by comparing both visual and quantitative changes. The images from the gastrointestinal samples suggest that Salmonella colonization was reduced as the MCFA concentration increased. Quantification of bioluminescence (photons/s) revealed a significant decrease for both the 4.5 and $6 \mathrm{lbs} /$ ton MCFA groups compared to the positive control group. In addition, the $6 \mathrm{lbs} /$ ton MCFA group 
had significantly lower bioluminescence than the $4.5 \mathrm{lbs} / \mathrm{ton}$ MCFA group, but there were no differences among the 1.5, 3, and $4.5 \mathrm{lbs} /$ ton MCFA groups. These data indicate that there was a dose-dependent decrease in Salmonella (Meckel's diverticulum to the cloaca) resulting from MCFA supplementation. Furthermore, when Salmonella was enumerated in the ceca, there was a significant decrease in Salmonella numbers for the $6 \mathrm{lbs} /$ ton MCFA group (1- $\log _{10} \mathrm{CFU} / \mathrm{g}$ reduction) compared to the positive control group. This study shows that MCFA can reduce early Salmonella colonization in turkeys and highlights the utility of bioluminescence imaging for evaluating treatment efficacy.

Several studies have evaluated the effect of MCFA on Salmonella colonization in poultry. Broilers fed diets containing caprylic acid (C8) at $0.7 \%$ (14 lbs/ton) and 1\% (20 lbs/ ton) had reduced Salmonella numbers in the gastrointestinal tract, liver, and spleen at 5-10 days PI (Kollanoor-Johny et al., 2009, 2012). Similarly, layers supplemented with caproic acid (C6) at $3 \mathrm{~g} / \mathrm{kg}$ of feed (6 lbs/ton) had lower Salmonella numbers in the ceca, liver, and spleen at 3 days PI (Van Immerseel et al., 2004a). In this study, we showed that turkeys provided a combination of MCFA (C6-C12) at 6 lbs/ ton of feed had reduced Salmonella colonization in the gastrointestinal tract at 3 days PI. All of the above treatments resulted in a $1-\log _{10} \mathrm{CFU} / \mathrm{g}$, or greater, reduction of Salmonella in the ceca. Collectively, these results indicate that MCFA decrease Salmonella colonization in poultry. Feed inclusion rates as low as $6 \mathrm{lbs} /$ ton appear to be effective and may further enhance the practicality of using such products in commercial poultry production.

\section{Conclusion}

For this study a bioluminescent Salmonella infection model was used for in vivo screening of a MCFA product in turkeys. Early colonization was evaluated because previous studies have indicated that treatment during this time may be particularly effective for controlling Salmonella within a flock. Birds were infected with bioluminescent Salmonella Typhimurium on day-of-hatch and provided feed containing MCFA ranging from 0 to $6 \mathrm{lbs} /$ ton. Significant decreases in both bioluminescence and Salmonella numbers (1-log 10 CFU/g reduction) were detected in the $6 \mathrm{lbs} / \mathrm{ton}$ MCFA treated poults at 3 days PI. This was the first study to evaluate the effect of MCFA on Salmonella in turkeys and these results indicate that MCFA supplementation can reduce early colonization. Further studies are needed to determine what impact MCFA treatments have on Salmonella colonization throughout the production cycle.

\section{Acknowledgments}

The authors thank Jason Regalado for his assistance in enumeration of Salmonella. This study was funded by PMI Nutritional Additives ${ }^{\mathrm{TM}}$.

\section{Disclosure Statement}

The MCFA product used in these studies is distributed in the United States by PMI Nutritional Additives ${ }^{\mathrm{TM}}$. No other competing financial interests exist.

\section{References}

Evans NP, Evans RD, Regalado J, Sullivan JF, Dutta V, Elvinger F, Pierson FW. Preharvest Salmonella detection for evaluation of fresh ground poultry product contamination. J Food Prot 2015;78:1266-1271.

Foucault M-L, Thomas L, Goussard S, Branchini BR, GrillotCourvalin C. In vivo bioluminescence imaging for the study of intestinal colonization by Escherichia coli in mice. Appl Environ Microbiol 2009;76:264-274.

Gast RK, Beard CW. Age-related changes in the persistence and pathogenicity of Salmonella Typhimurium in chicks. Poult Sci 1989;68:1454-1460.

Gast RK, Holt PS. Persistence of Salmonella enteritidis from one day of age until maturity in experimentally infected layer chickens. Poult Sci 1998;77:1759-1762.

Gould LH, Walsh KA, Vieira AR, Herman K, Williams IT, Hall AJ, Cole DJ. Surveillance for foodborne disease outbreaksUnited States, 1998-2008. Morb Mortal Wkly Rep 2013; $62: 1-34$.

Jackson BR, Griffin PM, Cole D, Walsh KA, Chai SJ. Outbreak-associated Salmonella enterica serotypes and food commodities, United States, 1998-2008. Emerg Infect Dis 2013;19:1239-1244.

Karsi A, Howe K, Kirkpatrick TB, Wills R, Bailey RH, Lawrence ML. Development of bioluminescent Salmonella strains for use in food safety. BMC Microbiol 2008;8:10.

Kollanoor-Johny A, Baskaran SA, Charles AS, Amalaradjou MAR, Darre MJ, Khan MI, Hoagland TA, Schreiber DT, Donoghue AM, Donoghue DJ, Venkitanarayanan K. Prophylactic supplementation of caprylic acid in feed reduces Salmonella Enteritidis colonization in commercial broiler chicks. J Food Prot 2009;72:722-727.

Kollanoor-Johny A, Mattson T, Baskaran SA, Amalaradjou MAR, Hoagland TA, Darre MJ, Khan MI, Schreiber DT, Donoghue AM, Donoghue DJ, Venkitanarayanan K. Caprylic acid reduces Salmonella Enteritidis populations in various segments of digestive tract and internal organs of 3- and 6week-old broiler chickens, therapeutically. Poult Sci 2012; 91:1686-1694.

Kwon YM, Ricke SC. Induction of acid resistance of Salmonella Typhimurium by exposure to short-chain fatty acids. Appl Environ Microbiol 1998;64:3458-3463.

Marin C, Lainez M. Salmonella detection in feces during broiler rearing and after live transport to the slaughterhouse. Poult Sci 2009;88:1999-2005.

Mulder RWAW. Impact of transport and related stresses on the incidence and extent of human pathogens in pigmeat and poultry. J Food Saf 1995;15:239-246.

Nakai SA, Siebert KJ. Validation of bacterial growth inhibition models based on molecular properties of organic acids. Int $\mathbf{J}$ Food Microbiol 2003;86:249-255.

Rasschaert G, Houf K, Godard C, Wildemauwe C, PastuszczakFrak M, De Zutter L. Contamination of carcasses with Salmonella during poultry slaughter. J Food Prot 2008;71: 146-152.

Scallan E, Hoekstra RM, Angulo FJ, Tauxe RV, Widdowson MA, Roy SL, Jones JL, Griffin PM. Foodborne illness acquired in the United States-Major pathogens. Emerg Infect Dis $2011 ; 17: 7-15$.

Seleem M, Ali M, Abd Al-Azeem MW, Boyle SM, Sriranganathan N. High-level heterologous gene expression in Ochrobactrum anthropi using an A-rich UP element. Appl Microbiol Biotechnol 2007a;73:1123-1127. 
Seleem M, Ali M, Al-Azeem MWA, Boyle SM, Sriranganathan N. Enhanced expression, detection and purification of recombinant proteins using RNA stem loop and tandem fusion tags. Appl Microbiol Biotechnol 2007b;75:1385-1392.

Seleem MN, Ali M, Boyle SM, Sriranganathan N. Reporter genes for real-time in vivo monitoring of Ochrobactrum anthropi infection. FEMS Microbiol Lett 2008a;286:124-129.

Seleem MN, Ali M, Boyle SM, Sriranganathan N. Vectors for enhanced gene expression and protein purification in Salmonella. Gene 2008b;421:95-98.

Skrivanova E, Marounek M, Benda V, Brezina P. Susceptibility of Escherichia coli, Salmonella sp. and Clostridium perfringens to organic acids and monolaurin. Vet Med 2006;51: 81-88.

Upadhyaya I, Upadhyay A, Yin H-B, Nair MS, Bhattaram VK, Karumathil D, Kollanoor-Johny A, Khan MI, Darre MJ, Curtis PA, Venkitanarayanan K. Reducing colonization and eggborne transmission of Salmonella Enteritidis in layer chickens by in-feed supplementation of caprylic acid. Foodborne Pathog Dis 2015;12:591-597.

Van Immerseel F, De Buck J, Boyen F, Bohez L, Pasmans F, Volf J, Sevcik M, Rychlik I, Haesebrouk F, Ducatelle R. Medium-chain fatty acids decrease colonization and invasion through hilA suppression shortly after infection of chickens with Salmonella enterica serovar Enteritidis. Appl Environ Microbiol 2004a;70:3582-3587.

Van Immerseel F, De Buck J, Pasmans F, Bohez L, Boyen F, Haesebrouck F, Ducatelle R. Intermittent long-term shedding and induction of carrier birds after infection of chickens early posthatch with a low or high dose of Salmonella Enteritidis. Poult Sci 2004b;83:1911-1916.

Van Immerseel F, De Buck J, Pasmans F, Velge P, Bottreau E, Fievez V, Haesebrouk F, Ducatelle R. Invasion of Salmonella enteritidis in avian intestinal epithelial cells in vitro is influenced by short-chain fatty acids. Int $\mathbf{J}$ Food Microbiol 2003;85:237-248.

Van Immerseel F, Russell JB, Flythe MD, Gantois I, Timbermont L, Pasmans F, Haesebrouck F, Ducatelle R. The use of organic acids to combat Salmonella in poultry: A mechanistic explanation of the efficacy. Avian Pathol 2006;35:182-188.

Address correspondence to: Nicholas P. Evans, PhD PMI Nutritional Additives ${ }^{\mathrm{TM}}$

1080 County Road F West, MS 5340 Shoreview, MN 55126

E-mail: nevans@landolakes.com 